\title{
Factive islands and interrogative logical triviality*
}

\author{
Hana Kalpak \\ Stockholm University
}

\begin{abstract}
Factive predicates can prevent $w h$-movement from the clauses they embed (Cinque 1990). This factive island effect has recently been analysed as resulting from pragmatic constraints on questions: $w h$-extraction is thought to be prohibited either because the resulting interrogative is systematically infelicitous (Schwarz \& Simonenko 2018b), or because it is systematically blocked by competing interrogative forms (Schwarz, Oshima \& Simonenko 2019). This paper notes empirical problems for both of these accounts, and offers an alternative explanation of factive islands in terms of IL-triviality, a novel extension of the notion of L-triviality (Gajewski 2002) to interrogative sentences.
\end{abstract}

Keywords: factive islands, meaning-driven unacceptability, triviality, wh-questions

\section{Introduction}

Factive predicates can create factive islands, prohibiting certain wh-extractions from a complement clause. For instance, subject extraction succeeds in (1a), featuring the non-factive think, but is unacceptable in (1b), featuring the factive know: ${ }^{1}$

(1) a. Who $i$ does Sam think $\left[t_{i}\right.$ is the tallest of the students]?

b. $* \mathrm{Who}_{i}$ does Sam know $\left[t_{i}\right.$ is the tallest of the students]?

While early literature on factive islands focus on the role of the embedding predicate (e.g., Cinque 1990, Rooryck 1992), later literature has noted that factivity is not generally sufficient to prohibit extraction (e.g., Szabolcsi \& Zwarts 1993, Oshima 2007). In (1b), the failure of wh-extraction also depends on the uniqueness of the predicate applied to the gapped element. If we substitute the definite description the tallest of the students for an indefinite description, extraction succeeds:

(2) Who ${ }_{i}$ does Sam know $\left[t_{i}\right.$ is a tall student $]$ ?

* For many valuable comments, I am grateful to Nadine Theiler, Anders Schoubye, the audience and reviewers of SALT 30, and the audience of the 2019 Principles of formal semantics workshop.

1 The judgments may vary with (among other things) one's tendency towards echo question readings and cleft readings; see Section 4.3. Anecdotally, the latter tendency appears strong for speakers of languages like Swedish, where clefted interrogatives are extremely common (Brandtler 2019).

C2020 Kalpak 
Given this, a factive island will here be taken to be an environment characterized by (i) a factive embedding predicate, and (ii) uniqueness of the questioned element, encoded for instance by the predicate applied to the trace in the embedded clause.

The apparent dependence on non-syntactic factors like factivity and uniqueness has made factive islands a central target for theories about "meaning-driven unacceptability" (Schwarz \& Simonenko 2018b): unacceptability that seems to derive from constraints on meaning rather than from constraints on form. ${ }^{2}$ As the earliest "meaning-driven" account, Szabolcsi \& Zwarts (1993) proposed that factive islands derive from a mismatch between the semantic type of the domain of the wh-word and the operation denoted by its scope. Abrusán (2014) gives several arguments against this, and instead proposes that factive island constructions have contradictory presuppositions, and therefore are out. However, her analysis requires that presuppositions from the scope of a $w h$-word project universally with respect to the restrictor (see Section 4), which is not always the case (Schwarz \& Simonenko 2018a).

More recently, Schwarz \& Simonenko (2018b) (building on Oshima 2007) propose that factive islands result from necessary infelicity: they are unacceptable because they can never be felicitously used. Schwarz et al. (2019) show that the infelicity analysis fails to extend to factive island effects in multiple wh-questions, and propose an alternative account in terms of necessary blocking. On this analysis, both single and multiple $w h$-constructions involving factive islands are unacceptable because they are systematically dispreferred to competing interrogative forms.

This paper argues that both the necessary infelicity and the necessary blocking account suffer from empirical over- and undergeneration problems. I propose that factive island constructions are instead unacceptable because they are IL-trivial: They never have more than one defined answer, regardless of how their non-logical terms are interpreted. The notion of IL-triviality is a novel complement to the increasingly influential notion of L-triviality, proposed by Gajewski (2002, 2009).

Like Schwarz \& Simonenko (2018b) and Schwarz et al. (2019), I will focus on factive island constructions where uniqueness follows from well-known mechanisms, like the presuppositions of the definite article (see (1b)). For a brief discussion of constructions in which establishing uniqueness requires more involved reasoning, like those involving extraction of how and why adjuncts, see Section 4.3.

a. $\quad\left\{\right.$ Why $\left._{i} / \mathrm{How}_{i}\right\}$ does Kim think that Max travelled to Rio $t_{i}$ ?

b. $*\left\{\right.$ Why $\left._{i} / \mathrm{How}_{i}\right\}$ does Kim know that Max travelled to Rio $t_{i}$ ?

The paper is structured as follows. Section 2 outlines the necessary infelicity and the necessary blocking accounts factive islands, and presents some problems for both.

2 By "unacceptability", I will generally mean the strong, ungrammaticality-like type of unacceptability that many speakers get with factive island violations, to distinguish from mere infelicity or oddness. 
Section 3 introduces Gajewski's notion of L-triviality, and proposes a complementary notion of IL-triviality defined for interrogative sentences. Section 4 uses this notion to give a new analysis of factive islands, and Section 5 concludes.

\section{Pragmatic accounts of factive islands}

Before introducing Schwarz \& Simonenko 2018b and Schwarz et al. 2019, I will summarize the basic assumptions these works make about the semantics of questions.

Both assume a Hamblin (Hamblin 1973) question semantics, according to which interrogative sentences denote sets of propositions, intuitively corresponding to the possible answers to the interrogative. For example, at a world $w$ with the individuals Sam $(s), \operatorname{Kim}(k)$, and Max $(m)$, the interrogative Who left? denotes the set in (4) (provided it is defined, see below):

$$
\llbracket \text { Who left } ? \rrbracket_{w}=\left\{\lambda w^{\prime} . \text { left }_{w^{\prime}}(x) \mid \operatorname{human}_{w}(x)\right\}=\left\{\begin{array}{l}
\lambda w^{\prime} ._{\mathbf{l e f t}_{w^{\prime}}(s),} \\
\lambda w^{\prime} . \mathbf{l e f t}_{w^{\prime}}(k), \\
\lambda w^{\prime} . \mathbf{l e f t}_{w^{\prime}}(m)
\end{array}\right\}
$$

I will refer to interrogative sentence denotations as questions. The intension of an interrogative is a thus a function from worlds to questions. This function may be partial, restricted to a set of possible worlds corresponding to presupposed information. For example, wh-interrogatives are standardly taken to trigger the existential presupposition that at least one of their answers is true (e.g., Dayal 1996):

$$
\text { Who left? } \stackrel{\text { presup }}{\Rightarrow} \text { Someone left. }
$$

Then the intension of (5) is a function from worlds where this (underlined) presupposition is true, to sets of propositions, each of which captures that a certain (atomic) individual left.

$$
\llbracket \text { Who left? } \rrbracket=\lambda w: \exists x\left(\operatorname{left}_{w}(x)\right) \cdot\left\{\lambda w^{\prime} \cdot \operatorname{left}_{w^{\prime}}(x) \mid \operatorname{human}_{w}(x)\right\}
$$

Answers can presuppose things, so the propositions contained in a question can also be partial. For instance, at a world $w$ where the interrogative Who left the room? is defined, its answers presuppose the existence of a unique (salient) room:

$$
\begin{aligned}
& \llbracket \text { Who left the room? } \rrbracket_{w} \\
& =\left\{\lambda w^{\prime}: \exists ! y\left(\operatorname{room}_{w^{\prime}}(y)\right) \cdot \operatorname{left}_{w^{\prime}}\left(\boldsymbol{l} y \cdot \mathbf{r o o m}_{w^{\prime}}(y)\right)(x) \mid \operatorname{human}_{w}(x)\right\}
\end{aligned}
$$

\subsection{Necessary infelicity}

Schwarz \& Simonenko (2018b) elaborate on ideas by Oshima (2007), and argue 
Factive Islands and IL-triviality

that factive islands result from necessary infelicity: constructions like (1b), repeated below, are unacceptable because they have jointly unsatisfiable felicity conditions.

${ }^{*} \mathrm{Who}_{i}$ does Sam know $t_{i}$ is the tallest of the students?

Felicity conditions are understood as relations between sentences, interpretations, and context sets (Stalnaker 1978); sets of possible worlds corresponding to the intersection of a common ground. Interrogative sentences are assumed to have the felicity conditions given below. I use $\operatorname{dom}\left(\llbracket \varphi \rrbracket_{I}\right)$ to denote the set of worlds where $\varphi$ is defined, with respect to the interpretation $I$.

Felicity conditions (adapted from Schwarz \& Simonenko 2018b). An interrogative sentence $Q$ is felicitous with respect to an interpretation $I$ and a context set $c$ only if (i) $c \subseteq \operatorname{dom}\left(\llbracket Q \rrbracket_{I}\right)$ (the presuppositions of $Q$ are entailed by $c$ ), and there is an answer $p \in \llbracket Q \rrbracket_{I}$ such that (ii) $c \subseteq \operatorname{dom}(p)$ (the presuppositions of $p$ are entailed by $c$ ), and (iii) $c \nsubseteq p$ ( $p$ is not entailed by $c$ ).

The combination of clauses (ii) and (iii) is called the answerability condition. On Schwarz \& Simonenko's account, the necessary infelicity of factive island constructions results from a clash between felicity condition (i) and the answerability condition: one cannot be satisfied without violating the other. The reasoning requires the assumption that $w h$-questions come with an existential presupposition, as in (8).

*Who does Sam know is the tallest of the students?

$\stackrel{\text { presup }}{\Rightarrow}$ Someone is such that Sam knows that they are the tallest of the students.

Granting this assumption, we can show that $(1 b) /(8)$ is necessarily infelicitous. For a reductio, suppose that $I=(M, w, g), c$ is an interpretation and context set, respectively, with respect to which (8) is felicitous. By clause (i), $c$ entails the existential presupposition of (8) with respect to $I$ :

(A) $\quad c \subseteq \llbracket \exists ! x\left(\right.$ tallest-student $(x) \& \operatorname{know}(s, x=\imath y \cdot \operatorname{tallest}-$ student $(y)) \rrbracket_{I}$

By clause (ii), $c$ must also entail the presupposition of at least one answer to (8) in $I$. In fact, it must entail the presupposition of exactly one answer: as indicated in (9), the answers to (8) have mutually incompatible presuppositions. Each answer to (8) presupposes for some individual $d$, that $d$ is the unique tallest student of Sam's.

(9) 【Who does Sam know is the tallest of the students? $\rrbracket_{I}$

$=\left\{\lambda w^{\prime}:\right.$ !tallest-student ${ }_{w^{\prime}}(d)$.

$\left.\operatorname{know}_{w^{\prime}}(s, d=\imath y \cdot \operatorname{tallest-student}(y)) \mid \operatorname{human}_{w}(d)\right\}$

('!tallest-student $(d)$ ' abbreviates 'tallest-student $(d) \& \forall y$ (tallest-student $(y) \leftrightarrow$ $y=d)^{\prime}$.) Say that $c$ entails the presupposition of the answer (10), so that (B) holds: 


$$
\lambda w^{\prime}: \underline{\text { tallest-student }}{ }_{w^{\prime}}(a) \cdot \mathbf{k n o w}_{w^{\prime}}(s, a=\imath y \cdot \text { tallest-student }(y))
$$

$$
c \subseteq \llbracket ! \text { tallest-student }(a) \rrbracket_{I}
$$

Now, the combination of (A) and (B) implies (C); that is, that $c$ entails (10).

$$
c \subseteq \llbracket ! \text { tallest-student }(a) \cdot \operatorname{know}(s, a=\imath y \cdot \operatorname{tallest-student}(y)) \rrbracket_{I}
$$

Thus the only answer defined in $c$ is also entailed by $c$, in violation of condition (iii). We have a contradiction, and can conclude that (8) is infelicitous with respect to $c$ and $I$. Since these were arbitrary, this shows that (8) is necessarily infelicitous. According to Schwarz \& Simonenko, this explains the unacceptability of (8) and other factive island constructions like it.

\subsection{Necessary blocking}

Schwarz, Oshima \& Simonenko (2019) show that the necessary infelicity account cannot explain factive islands that appear in multiple wh-questions, like (11a):
a. *Who knows [that Caesar was murdered where]?
b. Who thinks [that Caesar was murdered where]?
c. ?Who knows [that Caesar sent troops where]? (Schwarz et al. 2019)

The acceptability of (11b) indicates that the unacceptability of (11a) depends on the factivity of the embedding verb. Despite slight markedness, the acceptability of (11c) indicates that the unacceptability of (11a) also depends on the uniqueness of the predicate in the embedded clause: one (typically) cannot get murdered at more than one location, but one (typically) can send troops to more than one location.

Unlike single $w h$-questions involving factive island violations, constructions like (11a) can have answers with compatible presuppositions. For instance, consider an interpretation $I$ with two possible knowers, Sam $(s)$ and $\operatorname{Kim}(k)$, and two possible locations, Rome $(r)$ and Alexandria $(a)$. Then (11a) denotes a question with four answers, each of which shares its presupposition with another answer:

$$
\begin{aligned}
& \text { «Who knows that Caesar was murdered where? } \rrbracket_{I} \\
& =\left\{\begin{array}{l}
\lambda_{w}: \text { murdered-at }_{w}(r)(c) \cdot \mathbf{k n o w}_{w}(s, \text { murdered-at }(r)(c)), \\
\lambda_{w}: \overline{\text { murdered-at }}_{w}(r)(c) \cdot \mathbf{k n o w}_{w}(k, \text { murdered-at}(r)(c)), \\
\lambda w: \operatorname{murdered-at}_{w}(a)(c) \cdot \mathbf{k n o w}_{w}(s, \text { murdered-at }(a)(c)), \\
\lambda_{w}: \overline{\text { murdered-at }}_{w}(a)(c) \cdot \mathbf{k n o w}_{w}(k, \text { murdered-at }(a)(c))
\end{array}\right\}=\left\{\begin{array}{l}
r_{1}, \\
r_{2}, \\
a_{1}, \\
a_{2}
\end{array}\right\}
\end{aligned}
$$

This means that even a context where it is established that Someone knows where Caesar was murdered (satisfying condition (i)) can entail the presupposition of some 
answer (satisfying condition (ii)) without entailing that answer itself (satisfying condition (iii)). For instance, consider any $c$ where it is established that one of $r_{1}$ and $r_{2}$ are true, but not which one. Then $c$ entails that Caesar was murdered in Rome (satisfying condition (ii) w.r.t. I), that Sam or Kim knows this (satisfying condition (i)), but leaves open which one (satisfying condition (iii)). Thus (11a) is felicitous in $c$, meaning that its unacceptability cannot be attributed to necessary infelicity.

To improve on this (lack of) prediction, Schwarz et al. (2019) propose an alternative account of factive islands in terms of necessary blocking. On this account, factive island constructions are unacceptable because they are necessarily dispreferred to competing interrogative forms, according to the below definition.

Blocking (adapted from Schwarz et al. 2019). An interrogative sentence $Q$ is blocked by an interrogative sentence $Q^{\prime}$ with respect to $I, c$ just in case (i) for all $p \in \llbracket Q \rrbracket_{I}$ defined at $c, p \in \llbracket Q^{\prime} \rrbracket_{I}$, and (ii) $Q^{\prime}$ is syntactically simpler than $Q$ is, and/or (iii) $Q^{\prime}$ has stronger presuppositions than $Q$ (satisfied in $c$ ).

In other words, $Q^{\prime}$ blocks $Q$ if any (defined) answer to $Q$ is also an answer to $Q^{\prime}$, and $Q^{\prime}$ is less marked and/or presupposes more than $Q$, provided these presuppositions are actually satisfied (cf. Heim's (1991) Maximize presupposition!). A sentence is necessarily blocked if it is blocked with respect to all interpretation-context pairs $I, c$ where it felicitous (given Schwarz \& Simonenko's felicity conditions; Section 2.1).

Schwarz et al. show that both single and multiple wh-constructions involving factive island violations are necessarily blocked. Consider again (11a)/(13):

*Who knows that Caesar was murdered where?

By felicity condition (ii), if (13) is felicitous with respect to an interpretation $I$ and a context set $c$, then $c$ entails the presupposition of at least one answer to (13) in $I$. As exemplified in (12), the answers to (13) presuppose, for some location $l$, that $l$ is the location of Caesar's murder. Then by assumption $c$ entails, for some location $l$, that $l$ is the location of Caesar's murder. This makes the multiple wh-question (13) blocked by the single $w h$-question (14):

$$
\begin{aligned}
& \text { Who knows that Caesar was murdered in } l \text { ? } \\
& \stackrel{\text { presup }}{\Rightarrow} \text { Caesar was murdered in } l \text {. }
\end{aligned}
$$

The set of defined answers to (13) is always a subset of the set of answers to (14). Given the interpretation $I$ from (11a), (13) is felicitous in $c$ if $c$ entails either that Caesar was murdered in Rome, or that he was murdered in Alexandria. If the former, $l=$ Rome and $\llbracket(14) \rrbracket_{I}=\left\{r_{1}, r_{2}\right\}$; if the latter, $l=$ Alexandria and $\llbracket(14) \rrbracket_{I}=\left\{a_{1}, a_{2}\right\}$.

Moreover, the single $w h$-question (14) is intuitively less complex than the multiple $w h$-question (13). Since the condition for blocking requires a less complex form 
or stronger presuppositions, this suffices to establish that (13) is necessarily blocked by single $w h$-questions like (14). ${ }^{3}$ According to Schwarz et al., this explains the unacceptability of (13) and multiple $w h$-interrogatives like it. Similar reasoning to the above shows that single $w h$-questions like (8) is necessarily blocked by polar questions like (15b), where $x$ is the tallest person according to a given context set.
a. $* \mathrm{Who}_{i}$ does Sam know $t_{i}$ is the tallest of the students?
b. Does Sam know that $x$ is the tallest of the students?

\subsection{Problems for pragmatic accounts}

Both the infelicity analysis and the blocking analysis derive the unacceptability of factive island constructions from conditions on their felicitous use as matrix questions. These analyses therefore do not explain why the unacceptability persists when factive island constructions are not used as matrix questions. For instance, Oshima (2007) notes that the factive island construction (16a) is in no way improved by being embedded under an attitude verb, as in (16b):

a. *Why ${ }_{i}$ does Ken know [that Alice insulted Pat $t_{i}$ ]?

b. *John wonders [why ${ }_{i}$ Ken knows [that Alice insulted Pat $\left.t_{i}\right]$ ].

The declarative sentence (16b) is hardly subject to the answerability condition posited for (16a). To wit, a sentence like (17) can be felicitously used in a context where it is established that Alice insulted Pat (and did nothing else), even though this would violate the answerability condition of the embedded question.

(17) John wonders [what ${ }_{i}$ Ken knows [that Alice did $\left.\left.t_{i}\right]\right]$.

Thus, it seems like (16b) as a whole is not necessarily infelicitous: in theory, it can be felicitously used in any context where John believes that Ken knows why Alice insulted Pat (the presupposition triggered by wonder). This means that (16b) is also not necessarily blocked. A context set $c$ for (16b) can entail that Alice insulted Pat because $r$, without entailing that John believes this. Then $c$ does not satisfy the presuppositions of a construction like (18), which therefore cannot block (16b).

John wonders [whether Ken knows [that Alice insulted Pat because $r$ ]]. $\stackrel{\text { presup }}{\Rightarrow}$ John believes [that Alice insulted Pat because $r$ ].

This is a problem of undergeneration. Both pragmatic accounts also have prob-

3 Unlike Schwarz \& Simonenko (2018b), Schwarz et al. assume that wh-questions do not presuppose existence. Then (14), which felicitously presupposes that Caesar was murdered at $l$, is also presuppositionally stronger than (13), which on these assumptions presupposes nothing. 
Factive Islands and IL-triviality

lems of overgeneration. The infelicity analysis predicts that any interrogative with tautologous or contradictory answers, like (19), are out.

Who is [happy or not happy]?

While odd, (19) is hardly unacceptable-for one, it has rhetorical uses. However, (19) necessarily violates the answerability condition. This required for felicity of an interrogative $Q$ with respect to $I, c$, that there be some answer $p \in \llbracket Q \rrbracket_{I}$ not entailed by $c$. Since any individual is either happy or not happy, all $p \in \llbracket(19) \rrbracket_{I}$ are tautologies. A tautology is entailed by any $c$. Thus all $p \in \llbracket(19) \rrbracket_{I}$ are entailed by any $c$, and (19) is never answerable, and so is necessarily infelicitous.

In order to exclude cases like (19), Schwarz \& Simonenko (2018b: 269) suggest that necessary infelicity only yields unacceptability when it results from a conflict between multiple felicity conditions. This indeed distinguishes factive island constructions, where condition (i) clashes with the answerability condition, from (19), where the answerability condition itself is unsatisfiable. However, this reasoning seems fallacious: How can necessarily violating a conjunction of felicity conditions result in unacceptability, while necessarily violating one of the conjuncts does not? After all, the violation of a conjunct implies the violation of the conjunction.

(19) is also necessarily blocked, since it is never felicitous. Similarly, (20) is necessarily blocked by anything less complex or presuppositional, since none of its answers are defined: they each presuppose that someone has an impossible property.

Who do you know is [both happy and not happy]?

To sum up: While both pragmatic accounts cover standard factive island constructions, only the blocking account extends to factive islands in multiple $w h$-questions. Crucially, neither account can explain the unacceptability of factive island constructions outside of matrix questions, and both accounts overgenerate and predict that any interrogative with tautologous or contradictory answers is unacceptable on a par with factive island constructions. The next section will outline the prerequisites for an alternative account, aimed at overcoming the problems of the pragmatic accounts.

\section{Interrogative Logical Triviality}

Factive islands seem to arise due to constraints on meaning, rather than constraints on form. Many cases of unacceptability seem to be meaning-driven in this sense. For instance, there is a sharp difference in acceptability within the minimal pair in (21), and within the minimal pair in (22), but no relevant differences in form.

(21) There is $\{$ some/*every $\}$ book on the table. 
(22) I've read $\{$ *some/every $\}$ book but War and Peace.

What, then, is wrong the meanings of the starred examples? According to Zucchi (1995), the badness of *There is every book... is due to the felicity conditions of the sentence making incompatible demands. The felicity conditions of existential there-sentences require the context set to be compatible with the set of books being empty, but a felicitous use of every book requires the context set to entail that there is at least one book. Like the pragmatic analyses of factive islands, this assumes that unacceptability of the relevant strength can be driven by constraints that pertain to the felicity conditions of the sentence, and therefore would be subject to simliar problems with embeddability.

Several classic works in formal semantics (e.g., Barwise \& Cooper 1981; von Stechow 1984; von Fintel 1993) discuss an alternative possibility: that the starred examples in (21) and (22) are bad because they are logically trivial (tautologous or contradictory). Indeed, *There is every... is tautologous on the Barwise \& Cooper (1981) semantics for existential there-sentences, while *some ... but constructions are contradictory on von Fintel's (1993) semantics for exceptive but. As these works note, however, this is not sufficient to explain the severity of the unacceptability of these sentences, which to most people are not just odd, but unacceptable, often to the degree that they are perceived as ungrammatical. Many trivial sentences are clearly much more acceptable, as evidenced by their ability to be communicatively used:

a. Interviewer: Truth is truth ...

b. $\quad$. Giuliani: No, it isn't truth! Truth isn't truth. ${ }^{4}$

Gajewski $(2002,2009)$ proposed a principled way of distinguishing "pathologically" trivial (declarative) sentences, like *There is every... and *some ... but sentences (on the given semantics'), from ordinary trivial sentences, like the ones in (23). This section introduces his suggestion (subsection 3.1), and proposes a complementary means for distinguishing interrogative sentences with pathological meanings (3.2).

\subsection{L-triviality}

The key idea of Gajewski 2002, 2009 is that that the triviality of a sentence is pathological only if it is to a certain extent independent of the interpretation of the sentence' non-logical vocabulary. Then the sentence is L(ogically)-trivial, and is in virtue of this, Gajewski postulates, unacceptable or (perceived as) ungrammatical. ${ }^{5}$ The class of "(non-)logical" vocabulary is notoriously difficult to define formally (see e.g., Abrusán 2014: Ch. 6 for a relevant overview of attempts). Like Gajewski, I will

4 From this NBC interview, around 0.30 .

5 Gajewski calls L-trivial sentences ungrammatical, simply. 
rely on fairly innocent stipulations about individual expressions, broadly assuming that (i) logical expressions tend to be permutation invariant or closed-class, like every, not, and the copula; and (ii) non-logical expressions tend to be variant and/or open-class, like truth, good, and Sam.

Formally, a sentence is L-trivial just in case it is tautologous or contradictory in every interpretation in which it is defined, for a non-uniform substitution of its non-logical vocabulary. This is made precise by the following definitions:

Logical skeleton. A logical skeleton $\operatorname{LS}(\varphi)$ of an expression $\varphi$ is obtained by substituting every non-logical constituent $\alpha_{\tau}$ in $\varphi$ for a new type $\tau$ constant.

L-triviality. A sentence $\varphi$ is L-trivial if and only if, for any logical skeleton $\operatorname{LS}(\varphi)$ of $\varphi$ and any interpretation $I$ where $\operatorname{LS}(\varphi)$ is defined: $\llbracket \operatorname{LS}(\varphi) \rrbracket_{I}=1(0)$.

That is, a sentence is L-trivial just in case each of its logical skeletons are tautologous or contradictory, or equivalently (I invite the reader to convince themselves of this), in case one of them is. Note that any logical skeleton of (24) is contingent: for instance, the one indicated to the right is true whenever $\llbracket \mathbf{P} \rrbracket=\llbracket \mathbf{Q} \rrbracket$, and false otherwise.

(24) Truth is truth. $\stackrel{\text { LS }}{\longmapsto} \mathbf{P}$ is $\mathbf{Q}$.

Thus, (24) is not L-trivial. In contrast, the logical skeleton in (25) is trivial, assuming the semantics for existential there-sentences proposed by Barwise \& Cooper. According to this, $\llbracket$ There is every $\mathbf{P} \rrbracket$ is true given an interpretation $I$ and domain $D$ just in case $I(\mathbf{P}) \subseteq D$. Of course, this is the case for any $I, D$, so that (25) is L-trivial.

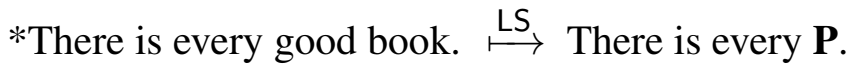

This explains the difference in acceptability between (24) and (25), given the following generalization (adapted from Gajewski 2002, 2009): If a sentence has an L-trivial constituent, then the sentence is unacceptable.

\subsection{IL-triviality}

The proposed connection between L-triviality and unacceptability has been used to explain a range of different semantic phenomena, such as NPI licensing (Chierchia 2013), degree islands (Abrusán 2014; Fox \& Hackl 2007), and selectional restrictions of clause-embedding verbs (Theiler, Roelofsen \& Aloni 2019; Mayr 2019). As a general source of meaning-driven unacceptability, however, L-triviality is insufficient: It cannot be used to explain patterns of unacceptability in sentences which do not express propositions, and thus lack truth-conditions. This excludes in particular 
interrogative sentences, which are taken to express sets of propositions (Hamblin 1973; Karttunen 1977) or partitions of logical space (Groenendijk \& Stokhof 1984).

Still, it would be surprising if logical skeletons with invariant denotations systematically yield unacceptability only for certain clause types. But what kind of invariant denotations could be pathological for interrogative sentences? Intuitively, the communicative usefulness of a declarative sentence derives at least in part from its ability to provide information: to express a proposition that can intersect with an information state to produce a non-empty, proper substate. Tautologous and contradictory sentences fail to do this, and are therefore communicatively deficient. Correspondingly, the communicative usefulness of an interrogative can be thought to come from its ability to disjoin information: to distinguish alternatives within a given information state. When an interrogative fails to do this-intuitively, if it has no more than one (defined) answer - it is communicatively deficient to a degree comparable to that of a tautologous or contradictory declarative. The following definition of I(nterrogative)L(ogical)-triviality implements the intuition that these cases are analogous, assuming a Hamblin 1973 question semantics. ${ }^{6}$

IL-triviality. An interrogative sentence $\varphi$ is $I L$-trivial iff, for any $\operatorname{LS}(\varphi)$ and interpretation $I$ where $\operatorname{LS}(\varphi)$ is defined: at most one $p \in \llbracket \operatorname{LS}(\varphi) \rrbracket_{I}$ is defined.

Corresponding to Gajewski's generalization, I propose that If a sentence has an IL-trivial constituent, then the sentence is unacceptable.

Unlike the pragmatic accounts discussed earlier, this will not predict unacceptability for constructions like (26). If (26) presupposes that Someone is happy and not happy, then it is never defined, making it necessarily infelicitous, and (trivially) necessarily blocked. However, it is not IL-trivial: The indicated logical skeleton can be defined and have multiple defined answers in any $I: \llbracket \mathbf{Q} \rrbracket_{I} \neq \llbracket \mathbf{S} \rrbracket_{I}$.

$$
\text { Who is [happy and not happy]? } \stackrel{\text { LS }}{\longmapsto} \text { Which } \mathbf{P} \text { is }[\mathbf{Q} \text { and not } \mathbf{S}] \text { ? }
$$

\section{Factive islands and IL-triviality}

I will now outline the full account of factive islands in terms of IL-triviality. After summarizing the assumptions to be made about the presuppositions of $w h$ interrogatives, I show that standard cases of factive islands are covered by the account (subsection 4.1), and that the analysis generalizes to factive islands in embedded and mutliple $w h$-interrogatives (subsection 4.2). After summarizing, I will also discuss an alternative definition of IL-triviality in terms of rescales, and briefly compare its merits to the definition in terms of logical skeletons (subsection 4.3).

6 In Möller Kalpak 2018, I proposed a corresponding definition of IL-triviality in inquisitive semantics. 
Factive Islands and IL-triviality

Like the necessary blocking analysis, but unlike the necessary infelicity analysis, the IL-triviality analysis will not depend on the assumption that $w h$-interrogatives presuppose that at least one of their answers is true (the existential presupposition). ${ }^{7}$ Like both pragmatic accounts, but unlike Abrusán's (2014) semantic account, the IL-triviality analysis will be independent of whether scope presuppositions are taken to project universally or existentially with respect to the wh-word's restrictor.

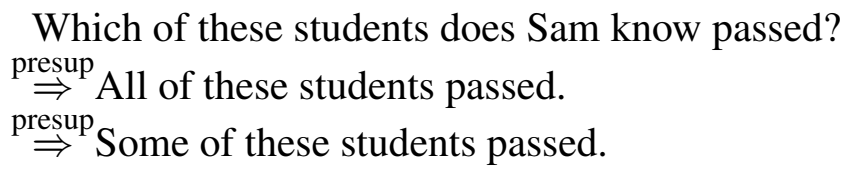

Universal projection

Existential projection

It will only be necessary to consider the presuppositions of things we want to show are not IL-trivial. If we can show that something is IL-trivial without assuming that it is presuppositional, we thereby show that it would be IL-trivial if presuppositional.

\subsection{Standard cases of factive islands}

We begin by ensuring that IL-triviality accurately predicts the unacceptability of "standard" (i.e., matrix, single $w h$-) factive island constructions, like (28).

*Who does Sam know is the tallest of the students?

For any interpretation $I=(M, w, g)$ where it is defined, (28) expresses (29):

【Who does Sam know is the tallest of the students? $\rrbracket_{I}$

$$
=\left\{\lambda w^{\prime}: \frac{\text { tallest-student }_{w^{\prime}}(d) \& \forall y\left(\text { tallest-student }_{w^{\prime}}(d) \leftrightarrow y=d\right) .}{\left.\operatorname{know}_{w^{\prime}}(s, d=\text { ly.tallest-student }(y)) \mid \operatorname{human}_{w}(d)\right\}} .\right.
$$

It is easy to see that this question can have at most one defined answer. Each answer presupposes, for some individual $d$, that $d$ is the unique tallest student. Of course at most one individual can be the unique tallest student in $I$, meaning that at most one answer can be defined: the one that agrees with $I$ on who the tallest student is.

In order to confirm (29) is IL-trivial, we need to show that its logical skeleton likewise has at most one defined answer. I will follow several previous authors (Del Pinal 2017; Theiler et al. 2019; Mayr 2019) in assuming that attitude verbs are generally decomposable into a purely logical component, responsible for presuppositions like factivity, and a non-logical component, encoding things like the verb's modal base. For instance, Del Pinal proposes that logical skeletons of know look like (30), which is just like ordinary know, but with an arbitrary accessibility relation

7 Note that holds only if the negative answer allowed in the absence of an existential presupposition is not treated as a semantic answer, i.e. is not taken to correspond to a proposition in the question. 
(here $\mathbf{R}$ ) instead of a specifically doxastic accessibility relation.

$$
\llbracket \operatorname{LS}(\text { know }) \rrbracket=\lambda p \lambda x \lambda w: p(w)=1 . \forall w^{\prime}\left[w^{\prime} \in \mathbf{R}_{w}^{x} \rightarrow p\left(w^{\prime}\right)=1\right]
$$

Given this, we obtain a logical skeleton of (28) by substituting the restrictor of the $w h$-word for an arbitrary predicate constant $\mathbf{Q}$, the proper name Sam for an individual constant $c$, the non-logical predicate tallest of the students for the predicate constant $\mathbf{P}$, and the attitude predicate know for a predicate $\operatorname{LS}(\mathbf{k n o w})$ :

*Who does Sam know is the tallest of the students?

$\stackrel{\text { LS }}{\longmapsto}$ Which $\mathbf{Q}$ does $c \mathrm{LS}(\mathbf{k n o w})$ is the $\mathbf{P}$ ?

For any interpretation $I=(M, w, g)$ where it is defined, this skeleton expresses (32):

$$
\begin{aligned}
& \llbracket \text { Which } \mathbf{Q} \text { does } c \operatorname{LS}(\mathbf{k n o w}) \text { is the } \mathbf{P} ? \rrbracket_{I} \\
& =\left\{\lambda w^{\prime}: \mathbf{P}_{w^{\prime}}(x) \& \forall y\left(\mathbf{P}_{w^{\prime}}(y) \leftrightarrow x=y\right)\right.
\end{aligned}
$$

Just as for (28), it is easy to see that at most one answer in (32) can be defined at a given world/interpretation. If $\llbracket \mathbf{Q} \rrbracket_{I}$ is a singleton, then (32) is, too; trivially making at most one answer defined. If $\llbracket \mathbf{Q} \rrbracket_{I}$ is not a singleton, then (32) contains exactly one answer for each $d: I(d) \in \llbracket \mathbf{Q} \rrbracket_{I}$, exactly one of which presupposes that

$$
\mathbf{P}(d) \& \forall y(\mathbf{P}(y) \leftrightarrow d=y) .
$$

Of course, only one such presupposition can be true at $I$ : only one individual $d$ can be the unique $\mathbf{P}$. Thus at most one answer to $\operatorname{LS}((28))$ can be defined at $I$, which, since $I$ was arbitrary, shows that (28) is IL-trivial.

Factivity. The IL-triviality of (28) depended on the factivity of the embedding predicate: A sentence like (33), featuring a non-factive predicate, is not IL-trivial.

Who does Sam think is the tallest of the students? $\stackrel{\text { presup }}{\Rightarrow}$ Sam believes that someone is the tallest of the students.

Without an embedding that presupposes the complement, the answers to (28) will not have mutually incompatible presuppositions. Consider an interpretation $I$ with the two individuals Sam $(s)$ and Alex $(a)$, one of which Sam thinks is the tallest student. Then (33) denotes a question with two non-presuppositional answers:

【Who does Sam think is the tallest of the students? II

$$
=\left\{\begin{array}{l}
\lambda w^{\prime} \cdot \text { believe }_{w^{\prime}}(s, ! \text { tallest-student }(s) \& s=\imath y \cdot \operatorname{tallest}(y)), \\
\lambda w^{\prime} \cdot \text { believe }_{w^{\prime}}(s, ! \text { tallest-student }(a) \& a=\imath y \cdot \operatorname{tallest}(y))
\end{array}\right\}
$$

This shows that (33) can have more than one defined answer, which entails that a 
Factive Islands and IL-triviality

logical skeleton of (33) likewise can have more than one defined answer. In other words, $I$ is a counterexample to the IL-triviality of (33).

Uniqueness. The IL-triviality of (28) likewise depends on the uniqueness of the predicate in the complement clause: (34) lacks this, and is therefore not IL-trivial.

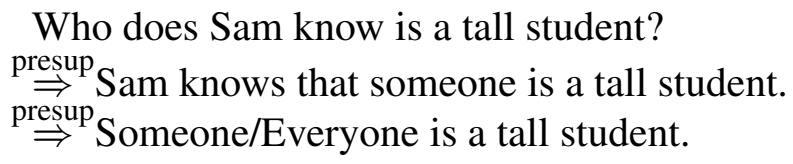

While the answers to (34) are generally presuppositional, the absence of uniqueness means that these presuppositions need not be mutually incompatible. Consider an interpretation $I$ with the two individuals Sam $(s)$ and Alex $(a)$, both of which are tall students according to $I$. Then (34) is defined and denotes a question with two answers, whose presuppositions are both defined:

«Who does Sam know is a tall student? $\rrbracket_{I}$

$$
=\left\{\begin{array}{l}
\lambda w^{\prime}: \operatorname{tall}_{w^{\prime}}(s) \& \operatorname{student}_{w^{\prime}}(s) \cdot \operatorname{know}_{w^{\prime}}(s, \operatorname{tall}(s) \& \operatorname{student}(s)), \\
\lambda w^{\prime}: \underline{\operatorname{tall}}_{w^{\prime}}(a) \& \operatorname{student}_{w^{\prime}}(a) \cdot \operatorname{know}_{w^{\prime}}(s, \operatorname{tall}(a) \& \operatorname{student}(a))
\end{array}\right\}
$$

This shows that (33), and by extension any $\operatorname{LS}((33))$, can have more than one defined answer, and so is not IL-trivial.

In conclusion, standard factive island constructions, like (28), are IL-trivial, and are so in virtue of featuring the particular combination of factivity and uniqueness characteristic of factive islands. Thus, the assumed connection between IL-triviality and (un)acceptability can explain the unacceptability of these constructions.

\subsection{Non-standard cases of factive islands}

An account of factive islands in terms of IL-triviality thus handles "standard" cases of factive islands just as well as the two pragmatic analyses discussed in Section 2 do. In addition, it extends more naturally than the pragmatic competitors to the noted cases of "non-standard" factive island constructions: those in non-matrix interrogatives, and those in multiple $w h$-interrogatives.

Factive islands in non-matrix clauses. Recall that the presence of an L-trivial constituent is generally sufficient for a sentence to be unacceptable. For instance, embeddings of the allegedly L-trivial (35a), like in (35b), are equally unacceptable:

a. *I've read some book but War and Peace.

b. *Sam knows [that I've read some book but War and Peace]. 
It is not immediately clear why unacceptability caused by L-triviality persists in this way. Unlike ill-formed constituents, which compositionally make any construction in which they appear uninterpretable, L-trivial constituents can occur in non-L-trivial sentences (viz., (35a) is contingent on the von Fintel 1993 semantics). Still, whatever will ultimately explains this attested behaviour of L-trivial sentences would likely predict the corresponding behaviour for IL-trivial sentences. Thus any sentence containing an IL-trivial construction is predicted to be unacceptable. In particular, the unacceptability of sentences containing factive island constructions, like (36), are explained by the IL-triviality of their embedded clause(s):

*Alex knows [who Sam knows is the tallest of the students].

Factive islands in multiple $\boldsymbol{w h}$-questions. Like the unacceptability caused by ill-formedness, but unlike that caused by infelicity, unacceptability caused by Ltriviality also seems to be preserved under $w h$-extraction. For instance, (37a) is not rendered acceptable through $w h$-clause formation as in (37b):

a. *I've read some book but War and Peace.

b. *Who $t_{i}$ read some book but War and Peace?

If IL-triviality is indeed a good correlate to L-triviality, we should expect it too to yield unacceptability that survives similar $w h$-extractions. This means in particular that multiple $w h$-interrogatives containing factive island violations are predicted to be unacceptable, in virtue of being derivable by extractions from IL-trivial constructions. For instance, the unacceptability of (38a) would be explained by the IL-triviality of (38b), since the former can be obtained from the latter by subject extraction.

a. *Who $t_{i} t_{\text {knows that }}$ who $_{j} t_{j}$ is the tallest of the students?

b. $* \mathrm{Who}_{j}$ does Sam know $t_{j}$ is the tallest of the students?

\subsection{Summary}

The table in Figure 4.3 summarizes the predictions of the IL-triviality account of factive islands, and compares it to the predictions of Schwarz \& Simonenko's (2018b) necessary infelicity account and Schwarz et al.'s (2019) necessary blocking account. As indicated, an account of factive islands in terms of IL-triviality not only makes the right predictions with respect to standard cases of factive islands, but also (by analogy to L-triviality) extends to predict the unacceptability of multiple wh- and non-matrix interrogatives containing factive island violations. Unlike both the necessary infelicity and the necessary blocking analysis, it does this without overgenerating to acceptable trivial questions like (39a) and (39b). As noted in Sec- 
Factive Islands and IL-triviality

\begin{tabular}{l|ccc} 
& Necessary infelicity & Necessary blocking & IL-triviality \\
\hline Standard FIs & $\checkmark$ & $\checkmark$ & $\checkmark$ \\
Multiple $w h$-FIs & $x$ & $\checkmark$ & $\checkmark$ \\
Non-matrix FIs & $x$ & $x$ & $\checkmark$ \\
Trivial questions & $\boldsymbol{x}$ & $\boldsymbol{x}$ & $\checkmark$
\end{tabular}

Figure 1 Summary of predictions.

tion 3.2, constructions like (26)/(39a) are not IL-trivial, and neither are constructions like (39b) (counterexample: any interpretation $I: \llbracket \mathbf{Q} \rrbracket_{I} \neq \llbracket \mathbf{S} \rrbracket_{I}$ ).

a. Who is [happy and not happy]?

b. Who do you know is both [happy and not happy]?

$\stackrel{\text { LS }}{\longmapsto}$ Which $\mathbf{P}$ does $c \operatorname{LS}($ know $)$ is $[\mathbf{Q}$ and not $\mathbf{S}]$ ?

Before concluding, I will briefly discuss (i) some factors that may obviate factive island effects, and (ii) the possibility of an alternative definition of IL-triviality.

A note on obviations. Sometimes, extraction from a factive island seems acceptable. For instance, certain additions of possibility modals seem to license extraction (Abrusán 2014), likely by eliminating uniqueness (Schwarz \& Simonenko 2018b). I will note two cases which are not previously discussed, but which could be explained on the present account. First, as suggested by Una Stojnić (p.c.), clefted $w h$-interrogatives seem more acceptable than their non-clefted counterparts:

(?)Who is it that Sam knows is the tallest of the students?

It-clefts are generally assumed to presuppose at most existence and exhaustivity (e.g., Büring \& Kriz 2013), approximated below:

It is $[\mathrm{Kim}]_{F}$ that Sam knows is the tallest student. $\stackrel{\text { presup }}{\Rightarrow}$ Someone is known by Sam to be the tallest student. $\stackrel{\text { presup }}{\Rightarrow}$ If Kim is known by Sam to be the tallest student, then nobody else is.

Clearly, the conjunction of these presuppositions would not contradict the corresponding presuppositions of, say, It is [Alex] $]_{F}$ that Sam knows is the tallest students. Thus the answers in a question denoted by (40) will have compatible presuppositions, meaning that it cannot be IL-trivial, and is not predicted to be unacceptable.

Unsurprisingly, factive island constructions are also improved by echo-question readings. The echo question (42a) is fine, while the interrogative in (42b) - where 
an echo question reading is blocked by the low focus-remains bad.

a. [Who $]_{F}$ does Sam know is the tallest of the students?

b. *Who does Sam know is [the tallest $]_{F}$ of the students?

On the present account, this pattern could be explained by adopting a metalinguistic account of echo questions (Sudo 2007). On such an account, echo questions-and presumably, also their answers - accommodate any presuppositions present on a non-echo reading. Then these answers are not presuppositional, and will always be defined. In this case (42a) is not IL-trivial, and thus not predicted to be unacceptable.

Skeletons or rescales? For reasons having to do with co-bound traces, Del Pinal (2017) argues that L-triviality should not be calculated with respect to logical skeletons, but instead with respect to rescales. To define these, let any RESCALE $\operatorname{Ri}_{i: i} \mathbb{N}$ be a subsective modifier (i.e., $\llbracket \operatorname{RESCALE}_{i}(\alpha) \rrbracket \subseteq \llbracket \alpha \rrbracket$ ), whose value otherwise varies with the interpretation. Then a rescale of a sentence $\phi$ is the result of replacing each minimal non-logical constituent token $\alpha$ in $\phi$ with $\operatorname{RESCALE}_{i}(\alpha)$, for a fresh $i$, viz.:

(43) Kim is tall and not tall.

Rescale: $\operatorname{ReSCALE}_{1}(\mathrm{Kim})$ is $\operatorname{RESCALE}_{2}$ (tall) and not $\operatorname{RESCALE}_{3}$ (tall).

We can implement this in the definitions of L-triviality and IL-triviality:

L-triviality II. A declarative sentence $S$ is $L$-trivial iff, for any rescale $S^{\prime}$ of $S$ and any interpretation $I$ where $S^{\prime}$ is defined: $\llbracket S^{\prime} \rrbracket_{I}=1(0)$.

IL-triviality II. An interrogative sentence $Q$ is $I L$-trivial iff, for any rescale $Q^{\prime}$ of $Q$ and interpretation $I$ where $Q^{\prime}$ is defined: at most one $p \in \llbracket Q^{\prime} \rrbracket_{I}$ is defined.

With respect to factive islands, a definition of IL-triviality in terms of rescales has potential advantages over a definition in terms of logical skeletons. First, recall the examples of factive island constructions with how and why from the introduction:

$*\left\{\right.$ Why $\left._{i} / \mathrm{How}_{i}\right\}$ does Kim know that Max travelled to Rio $t_{i}$ ?

Oshima (2007) and Schwarz \& Simonenko (2018b) argue that the presuppositions of the answers to (44) are indeed mutually incompatible, but that this is a result of them concerning reasons and manners. In the logical skeletons of (44), the restrictors of the $w h$-words are swapped for arbitrary constants. Thus, these skeletons will not always be interpreted as why- or how-questions. In contrast, the rescales of (44) will be interpreted as such, since they only involve semantic narrowing of the original restrictors. Thus (44) would only count as IL-trivial on IL-triviality II. 
Factive Islands and IL-triviality

Relatedly, Oshima (2007) and Schwarz \& Simonenko (2018b) argue that the questioned element in a factive island construction need not be unique by logical necessity. For instance, it is logically possible to be murdered at more than one location, yet (45) is unacceptable (according to Schwarz et al. 2019):

$$
\text { *Where }{ }_{i} \text { does Max know [that Caesar was murdered } t_{i} \text { ]? }
$$

However, the location queried by (45) is unique with respect to what Schwarz et al. call the accessible interpretations: roughly, interpretations that are especially normal or typical, like (arguably) the ones where one does not get murdered at more than one place. With respect only to such interpretations, (45) is IL-trivial according to the definition using rescales, but not according to the one using logical skeletons. A rescale of (45) will still express a question about a location of murder, while a logical skeleton does not even express the queried individual need not even be a location.

It is not clear exactly what determines (or should determine) which interpretations are "accessible", making a relativization of (I)L-triviality to such contexts a noninnocent move. For instance, can the set of accessible interpretations, and thereby the set of (I)L-trivial sentences, vary with context? Then we seem to be tracking a type of unacceptability quite different from the one originally targeted by Gajewski's L-triviality. ${ }^{8}$ A more conservative explanation may be given by noting that it seems particularly easy to mistake sentences like (45) for IL-trivial sentences during an on-line assessment. Some types of interpretations will intuitively be easier to access in a certain context; these will be the ones that we use to assess (I)L-triviality, but not the ones that actually determine it. Further investigation of this option, as well as a more thorough comparison between definitions, must be left to future work.

\section{Conclusion}

This paper argued that the unacceptability of factive island constructions stems from a semantic deficiency: factive island constructions sound bad because they are ILtrivial. If this is correct, the analysis of factive islands does not require the assumption that there are any essentially pragmatic sources of perceived ungrammaticality in natural language, pace Schwarz \& Simonenko (2018b); Schwarz et al. (2019). This does not exclude the possibility or usefulness of an external pragmatic rationale for the assumed connection between (I)L-triviality and perceived ungrammaticality. Yet, the predictive differences between the analysis proposed here and the pragmatic analyses point to the value of keeping such a rationale separate from the core explanatory features of the analysis of factive islands.

8 Thanks to Paolo Santorio for discussing this point with me. 


\section{References}

Abrusán, Márta. 2014. Weak island semantics. Oxford University Press. doi:10.1093/acprof:oso/9780199639380.001.0001.

Barwise, Jon \& Robin Cooper. 1981. Generalized quantifiers and natural language. Linguistics and Philosophy 4(2). 159-219. doi:10.1007/978-94-009-2727-8_10.

Brandtler, Johan. 2019. The question of form in the forming of questions: The meaning and use of clefted wh-interrogatives in Swedish. Journal of Linguistics 55(4). 755-794. doi:10.1017/S0022226718000634.

Büring, Daniel \& Manuel Kriz. 2013. It's that, and that's it! Exhaustivity and homogeneity presuppositions in clefts (and definites). Semantics and Pragmatics 6. 1-29. doi:10.3765/sp.6.6.

Chierchia, Gennaro. 2013. Logic in grammar: Polarity, free choice, and intervention. Oxford University Press. doi:10.1093/acprof:oso/9780199697977.001.0001.

Cinque, Guglielmo. 1990. Types of $\bar{A}$-dependencies. Cambridge, MA: MIT press.

Dayal, Veneeta. 1996. Locality in wh-quantification: Questions and relative clauses in Hindi. Dordrecht: Kluwer Academic Publishers. doi:10.1007/978-94-0114808-5_6.

Del Pinal, Guillermo. 2017. The logicality of language: A new take on triviality, ungrammaticality, and Logical Form. Nô̂s 53(4). doi:10.1111/nous.12235.

von Fintel, Kai. 1993. Exceptive constructions. Natural Language Semantics 1(2). 123-148. doi:10.1007/BF00372560.

Fox, Danny \& Martin Hackl. 2007. The universal density of measurement. Linguistics and Philosophy 29(5). 537-586. doi:10.1007/s10988-006-9004-4.

Gajewski, Jon. 2002. L-analyticity and natural language. Manuscript, MIT.

Gajewski, Jon. 2009. L-triviality and grammar. Handout of a talk delivered at the UConn Logic Group.

Groenendijk, Jeroen \& Martin Stokhof. 1984. On the semantics of questions and the pragmatics of answers: University of Amsterdam $\mathrm{PhD}$ dissertation.

Hamblin, Charles L. 1973. Questions in Montague grammar. Foundations of language 10(1). 41-53.

Heim, Irene. 1991. Artikel und Definitheit. Semantik: Ein internationales Handbuch der zeitgenössischen Forschung 487-535.

Karttunen, Lauri. 1977. Syntax and semantics of questions. Linguistics and philosophy 1(1). 3-44. doi:10.1007/BF00351935.

Mayr, Clemens. 2019. Triviality and interrogative embedding: Context sensitivity, factivity, and neg-raising. Natural Language Semantics 27(3). 227-278. doi:10.1007/s11050-019-09153-8.

Möller Kalpak, Hana. 2018. Inquisitive logical triviality and grammar. University of Amsterdam Master's thesis. 
Factive Islands and IL-triviality

Oshima, David Y. 2007. On factive islands: Pragmatic anomaly vs. pragmatic infelicity. Japanese Society for Artificial Intelligence (JSAI) 147-161. doi:10.1007/9783-540-69902-6_14.

Rooryck, Johan. 1992. Negative and factive islands revisited. Journal of Linguistics 28(2). 343-374. doi:10.1017/S0022226700015255.

Schwarz, Bernhard, David Yoshikazu Oshima \& Alexandra Simonenko. 2019. Factive islands from necessary blocking. Semantics and Linguistic Theory (SALT) 29. 529-548. doi:10.3765/salt.v29i0.4628.

Schwarz, Bernhard \& Alexandra Simonenko. 2018a. Decomposing universal projection in questions. Sinn und Bedeutung $(\mathrm{SuB})$ 22. 361-374.

Schwarz, Bernhard \& Alexandra Simonenko. 2018b. Factive islands and meaningdriven unacceptability. Natural Language Semantics 26(3-4). 253-279. doi:10.1007/s11050-018-9146-2.

Stalnaker, Robert C. 1978. Assertion. In P. Cole (ed.), Pragmatics, 315-332. New York Academic Press. doi:10.1163/9789004368873_013.

von Stechow, Arnim. 1984. Comparing semantic theories of comparison. Journal of semantics 3(1-2). 1-77. doi:10.1093/jos/3.1-2.1.

Sudo, Yasutada. 2007. A metalinguistic semantics for echo questions. Amsterdam Colloquium (AC) 16. 205-211.

Szabolcsi, Anna \& Frans Zwarts. 1993. Weak islands and an algebraic semantics for scope taking. Natural language semantics 1(3). 235-284. doi:10.1007/BF00263545.

Theiler, Nadine, Floris Roelofsen \& Maria Aloni. 2019. Picky predicates: Why 'believe' doesn't like interrogative complements, and other puzzles. Natural Language Semantics 27(2). 95-134. doi:10.1007/s11050-019-09152-9.

Zucchi, Alessandro. 1995. The ingredients of definiteness and the definiteness effect. Natural Language Semantics 3(1). 33-78. doi:10.1007/BF01252884.

Hana Kalpak

Department of Philosophy

Stockholm University

hana.kalpak@philosophy.su.se 